\title{
Application of adjoint Monte Carlo to accelerate simulations of mono-directional beams in treatment planning for Boron Neutron Capture Therapy
}

\author{
V. A. Nievaart ${ }^{a}$ \\ Department of Applied Sciences, Delft University of Technology, Lorentzweg 1, \\ 2628CJ Delft, The Netherlands and Joint Research Centre of the European Commission, P.O. Box 2, \\ $1755 Z G$ Petten, The Netherlands \\ D. Légràdy \\ Department of Applied Sciences, Delft University of Technology, Lorentzweg 1, \\ 2628CJ Delft, The Netherlands \\ R. L. Moss \\ Joint Research Centre of the European Commission, P.O. Box 2, 1755ZG Petten, The Netherlands
}

J. L. Kloosterman

Department of Applied Sciences, Delft University of Technology, Lorentzweg 1, 2628CJ Delft, The Netherlands

T. H. J. J. van der Hagen

Department of Applied Sciences, Delft University of Technology, Lorentzweg 1, 2628CJ Delft, The Netherlands and Reactor Institute Delft, Delft University Technology, Mekelweg 15, 2629JB Delft, The Netherlands

\section{H. van Dam}

Department of Applied Sciences, Delft University of Technology, Lorentzweg 1, 2628CJ Delft, The Netherlands

(Received 12 May 2006; revised 8 February 2007; accepted for publication 8 February 2007; published 16 March 2007)

This paper deals with the application of the adjoint transport theory in order to optimize Monte Carlo based radiotherapy treatment planning. The technique is applied to Boron Neutron Capture Therapy where most often mixed beams of neutrons and gammas are involved. In normal forward Monte Carlo simulations the particles start at a source and lose energy as they travel towards the region of interest, i.e., the designated point of detection. Conversely, with adjoint Monte Carlo simulations, the so-called adjoint particles start at the region of interest and gain energy as they travel towards the source where they are detected. In this respect, the particles travel backwards and the real source and real detector become the adjoint detector and adjoint source, respectively. At the adjoint detector, an adjoint function is obtained with which numerically the same result, e.g., dose or flux in the tumor, can be derived as with forward Monte Carlo. In many cases, the adjoint method is more efficient and by that is much quicker when, for example, the response in the tumor or organ at risk for many locations and orientations of the treatment beam around the patient is required. However, a problem occurs when the treatment beam is mono-directional as the probability of detecting adjoint Monte Carlo particles traversing the beam exit (detector plane in adjoint mode) in the negative direction of the incident beam is zero. This problem is addressed here and solved first with the use of next event estimators and second with the application of a Legendre expansion technique of the angular adjoint function. In the first approach, adjoint particles are tracked deterministically through a tube to a (adjoint) point detector far away from the geometric model. The adjoint particles will traverse the disk shaped entrance of this tube (the beam exit in the actual geometry) perpendicularly. This method is slow whenever many events are involved that are not contributing to the point detector, e.g., neutrons in a scattering medium. In the second approach, adjoint particles that traverse an adjoint shaped detector plane are used to estimate the Legendre coefficients for expansion of the angular adjoint function. This provides an estimate of the adjoint function for the direction normal to the detector plane. In a realistic head model, as described in this paper, which is surrounded by 1020 mono-directional neutron/gamma beams and from which the best ones are to be selected, the example calculates the neutron and gamma fluxes in ten tumors and ten organs at risk. For small diameter beams $(5 \mathrm{~cm})$, and with comparable relative errors, forward Monte Carlo is seen to be 1.5 times faster than the adjoint Monte Carlo techniques. For larger diameter neutron beams (10 and $15 \mathrm{~cm}$ ), the Legendre technique is found to be 6 and 20 times 
faster, respectively. In the case of gammas alone, for the 10 and $15 \mathrm{~cm}$ diam beams, both adjoint Monte Carlo Legendre and point detector techniques are respectively 2 and 3 times faster than forward Monte Carlo. (C) 2007 American Association of Physicists in Medicine.

[DOI: $10.1118 / 1.2712573$ ]

Key words: adjoint Monte Carlo, mono-directional beams, treatment planning, BNCT

\section{INTRODUCTION}

In current radiotherapy treatment planning, the treatment planner has first to define certain parameters such as the beam characteristics, the number of beams to be used, their position and orientation, together with the target volume and the organs at risk (OAR). The next step is to make a treatment plan by adjusting the weights and/or shapes of these selected beams such that the target volume receives the required (high) dose without exceeding the tolerance doses of the OAR. Concerning the first step, it would already be an improvement if the optimal beams (location, direction, energy, and shape) were selected automatically. This is, in principle, possible with the application of adjoint transport theory. A detailed theoretical description of the adjoint method is not provided in this paper and the reader is referred to other literature. ${ }^{1-3}$ Furthermore, this paper deals with radiotherapy treatment planning in the field of Boron Neutron Capture Therapy (BNCT). A brief description of BNCT is given in Sec. II A. At this stage it is sufficient to know that BNCT patients are irradiated with a mixed radiation beam of neutrons and gammas and that treatment plans can only be made with Monte Carlo (MC) based programs. In normal MC transport calculations the particles start at a source, lose energy while traveling, before they are registered when passing a detector. This MC mode is referred to as forward MC and implies that forward particles travel from the forward source to the forward detector. This way of traveling can be reversed and is referred to as adjoint MC. Socalled adjoint particles start at the forward detector and travel backwards, gaining energy before they are registered when passing the forward source. Actually, in adjoint MC the forward detector becomes the adjoint source and the forward source becomes the adjoint detector. (In the remaining text, when the single word "source," "detector," or "particle" is written, the source, detector, or particle in the real world is meant. With the additive "forward," the same source, detector or particle is suggested but then in the simulated world. While there is practically no difference, "forward" is sometimes omitted in the text in order to improve the readability. The additive "adjoint" is always written whenever simulated adjoint particles travel from the adjoint source to the adjoint detector.) With the adjoint method the user obtains at the adjoint detector the adjoint function which represents after the definition given by Bell and Glasstone, ${ }^{4}$ a measure of the "importance" of a forward source particle in contributing to the response of the forward detector. The adjoint function will be further discussed in Sec. II B. For treatment planning, the adjoint function opens the possibility to gather information about the position, angle, and energy of beam particles that contribute to tumors and OAR. Instead of performing a great number of forward calculations to find the optimal irradiation conditions, the adjoint can give these in a few calculations. The adjoint approach is advantageous when the number of beam exits (adjoint detectors) is much greater than the number of tumors and OAR (adjoint sources). The actual advantage depends on the number and size of the tumors and OAR and on the number and size of the beam exits.

As stated in an article by Wang et al., ${ }^{5}$ Goldstein and Difilippo $^{6-8}$ can be regarded as the pioneers of the application of adjoint $\mathrm{MC}$ in treatment planning. Although they and also others ${ }^{9,10}$ have proven that it is applicable, the adjoint MC method is still not widely used in the clinical community.

Most of the published works in this field use the Monte Carlo N-particle transport code system MCNP from Los Alamos National Laboratory. ${ }^{11}$ Furthermore, in the simulation geometries, the irradiated bodies are all surrounded by a spherical or cylindrical surface, which is segmented to form adjoint detector areas. Such a set-up implies that the adjoint functions are determined at fixed positions during a MCNP calculation. The adjoint detector segments can have the shape of the real physical beam or form a lattice of smaller adjoint detectors representing an array of pencil beams. This last approach would be preferable if the lattice is fine structured in size, energy, and angular bins. Only then would a superposition of the pencil beams, to represent a real beam opening, make sense. Even with present day MC codes and computer power, this fine structure approach is simply impossible due to the time required to achieve decent statistics. At present, rather large lattices need to be used, which can provide no more than a coarse grid of optimal directions to irradiate from. In a publication of Jeraj and Keall, ${ }^{10}$ it is shown that this approach, used as a first optimization step, is already a great improvement for the treatment plan.

Nevertheless, real beam shaped adjoint detector segments provide an exact value of the contribution for a certain beam. This article deals only with the real beam shaped adjoint detector segments and addresses how to overcome its associated problem. Before explaining this problem, it has to be explained first that only adjoint particles at the adjoint detector with favorable phase space coordinates are useful. If the treatment beam has for instance an angular divergence of 2 degrees, only adjoint particles hitting the adjoint detector plane within $2^{\circ}$ to the normal are valuable. Note that these adjoint particles travel in the opposite direction of the particles in the treatment beam. Practically, it will take a long time before reasonable statistics are obtained for a specific (narrow) range of phase space coordinates. Such an approach 
is applied by Wang et al. ${ }^{5}$ Whenever the angular divergence of the treatment beam is zero and, thus, the particles leave the beam exit plane perpendicularly, the calculation will take infinitely long. This is due to the fact that the possibility of an adjoint particle hitting the adjoint detector plane (=beam exit plane) perpendicularly is virtually zero. It is for these so-called mono-directional treatment beams that in this work two methods are developed and applied to enable adjoint MC for treatment planning.

The first method is based on next event estimators and the second method is based on the expansion of the adjoint function in Legendre polynomials in the MC environment. The second method originates from the functional expansion technique (FET) described by Beers and Pine. ${ }^{12}$ By applying Legendre expansion in MC treatment planning, all adjoint particles can contribute to provide a good estimate for the contribution of the mono-directional beam to a tumor or OAR. As a result, the adjoint function of a certain angle can be calculated much more quickly everywhere around the geometry of a patient.

\section{MATERIALS AND METHODS}

This section starts by giving more information about $\mathrm{BNCT}$, adjoint $\mathrm{MC}$, the adjoint function and its relation with the forward results. In Sec. II C, the next event estimator approach is described and in Sections II D-II G, the FET with Legendre polynomials are described. In Sections III and IV, the two developed methods are demonstrated by two examples. The first example shows the validity of the two adjoint MC methods and describes the convergence properties of the Legendre technique. In the second example, a human head phantom is irradiated with mono-directional neutron and gamma beams and it shows the cases where the adjoint $\mathrm{MC}$ technique is preferred over normal forward MC in terms of calculation speed. Finally, the optimum positions and orientations of a neutron beam are presented which may also be regarded as an introductory step for further investigations in the optimization of treatment planning.

\section{A. Brief description of Boron Neutron Capture Therapy (BNCT)}

Boron Neutron Capture Therapy (BNCT) requires that thermal neutrons are delivered at sites of cancer cells, containing much more boron- $10\left({ }^{10} \mathrm{~B}\right)$ than the surrounding healthy cells. As a result, due to the reaction of neutrons with ${ }^{10} \mathrm{~B}$, high energy particles are produced that, in principle, can kill each cancer cell. Consequently, healthy cells containing no ${ }^{10} \mathrm{~B}$ receive lower doses and will be spared. This nonboron dose is a result of the thermal neutrons creating secondary particles due to hydrogen and nitrogen reactions in the tissue. This could give an unwanted dose in several OAR, which limits the irradiation time. In BNCT, patients are most often irradiated with epithermal source neutrons, as the neutrons then thermalize due to interactions with intermediate tissues before reaching the tumor. The aim is to have a lot of thermal neutrons at the location of the cancer. In reactor based BNCT, as in Petten (The Netherlands), there is a cer- tain dose coming from the gammas originating from the reactor core. It is therefore necessary to perform the calculations also for this type of irradiation. Since neutrons and gammas are both important in BNCT, the adjoint techniques presented in this paper will be demonstrated for both types of irradiation. More details on BNCT can be found in many publications. ${ }^{13,14}$

\section{B. Relation between forward flux and adjoint function}

In general, the adjoint method is preferable when the forward detector is small and the forward source large. Adjoint particles that start at a small forward detector have a larger probability to be detected at the forward source, from which a statistically reliable "signal" (the adjoint function) can be obtained. In treatment planning the adjoint particles start at the tumor or at the OAR and have to be detected everywhere outside the body in order to find the optimum beam location and orientation. Consequently, not one large adjoint detector, but many smaller ones, have to be modeled. The forward and adjoint solutions are called the forward neutron or gamma flux $\phi(\underline{r}, \underline{\Omega}, E)$ and the adjoint function $\phi^{+}(\underline{r}, \underline{\Omega}, E)$, respectively. Both quantities are a function of the position $\underline{r}$, the direction $\Omega$ and the energy $E$ of the (adjoint) particles of interest. From Williams and Engle ${ }^{15} \phi$ may be imagined as a quantity related to the "particle distribution," whilst $\phi^{+}$can be interpreted as the "effect distribution" (expected detector response). The particle transport equation for neutrons and photons is based on the linear Boltzmann equation. In the time-independent form, a shorthand notation of this equation can be written by introducing a transport operator $L$. This operator includes the characteristic streaming, loss, and scattering terms operating on the flux $\phi$. Conversely, an adjoint transport equation can be defined with a mathematically adjoint transport operator $L^{+}$operating on $\phi^{+}$. Before presenting the important relation between the forward flux $\phi$ and adjoint function $\phi^{+}$, the forward and adjoint transport equations are presented, with accompanying boundary conditions. Using a shorthand notation after Bell and Glasstone, ${ }^{4}$ the relevant forward problem for $\phi$ discussed in this paper is defined by

$$
\begin{aligned}
& L \phi(\underline{r}, \underline{\Omega}, E)=0, \quad \underline{r} \in V, \\
& \phi(\underline{r}, \underline{\Omega}, E)=\phi^{b}(\underline{r}, \underline{\Omega}, E), \quad \underline{r} \in S, \underline{\Omega} \cdot \hat{n}<0,
\end{aligned}
$$

where $\phi^{b}$ is a prescribed forward boundary source and $S$, of which $\hat{n}$ is the unit outer normal vector, is the boundary surface of volume $V . L$ is the forward transport operator. In this paper all treated problems have a prescribed forward boundary source $\phi^{b}$ of particles crossing $S$ in the inward direction. The relevant adjoint problem for $\phi^{+}$satisfies

$$
\begin{gathered}
L^{+} \phi^{+}(\underline{r}, \underline{\Omega}, E)=-\Sigma_{d}(\underline{r}, E), \quad \underline{r} \in V, \\
\phi^{+}(\underline{r}, \underline{\Omega}, E)=0, \quad \underline{r} \in S, \underline{\Omega} \cdot \hat{n}>0,
\end{gathered}
$$

where $L^{+}$is the adjoint transport operator and $\Sigma_{d}$ is the detector response function. The latter is usually given by a macroscopic cross section and defines in the adjoint calcula- 
tion the source of the adjoint particles. Equations (1)-(4) illustrate that the "forward solution" is related to a specific boundary source and the "adjoint solution" involves a specific detector $\Sigma_{d}$. The relation between $\phi$ and $\phi^{+}$is given by

$$
\begin{aligned}
& \int_{V} \int_{4 \pi} \int_{0}^{\infty} \Sigma_{d}(\underline{r}, E) \phi(\underline{r}, \underline{\Omega}, E) d E d \Omega d V \\
& \quad=\int_{S} \int_{\Omega \cdot \hat{n}<0} \int_{0}^{\infty}|\underline{\Omega} \cdot \hat{n}| \phi^{+}(\underline{r}, \underline{\Omega}, E) \phi^{b}(\underline{r}, \underline{\Omega}, E) d E d \Omega d S,
\end{aligned}
$$

which can be derived following the literature on the adjoint technique as written in the Introduction. Interpreted for treatment planning, Eq. (5) tells that the integral of the adjoint function multiplied with the appropriate forward boundary source function over the surface $S$ of volume $V$, in which the beam exit is located, all solid angles $\Omega$ pointing inward volume $V$, and all energies $E$, gives the left-hand side of Eq. (5), the total detector response. This total detector response is the contribution of the treatment beam to the flux or dose in, for example, a tumor. This is when the adjoint source is located in this tumor with a source spectrum $\Sigma_{d}$ according to the flux or dose. Since in this paper the focus is on incident beams that are completely or nearly mono-directional, the boundary source becomes

$$
\phi^{b}(\underline{r}, \underline{\Omega}, E) \approx \Phi^{b}(\underline{r}, E) \delta\left(\underline{\Omega}-\underline{\Omega}_{0}\right),
$$

where $\delta$ is the Dirac-function and $\Omega_{0}$ is the direction of the particles in the beam.

MCNP tracks adjoint neutrons, as well as adjoint gammas, but the adjoint MC implemented here is restricted to using multigroup cross section libraries. The multigroup libraries are problem dependent. It should be noted that MCNP adjoint results have to be normalized to the real treatment source. This normalizing is described by Wagner et al. ${ }^{16}$ and Difilippo. ${ }^{6}$ A thorough description of adjoint MC for neutrons can be found in Lewis and Miller ${ }^{17}$ and Hoogenboom. ${ }^{18}$

Although perhaps confusing, the reader has to be informed that it is possible to calculate adjoint functions with forward MC. This possibility is applied in Example I (Sec. III). The calculation of the energy dependent adjoint function in forward mode is performed by adding at the forward detector all those scores of the forward particles which started from the same energy group at the source. Plotting all these sums for each source energy group in a graph gives the adjoint function at the source location. This method is similar to the definition of the contribution function which is defined as describing the contribution of source particles to the detector. ${ }^{4}$ Of course, it is still a forward MC calculation and, therefore, the statistical uncertainties of an estimate can only improve when a forward particle reaches the detector.

\section{Next event estimator approach: The adjoint point detector technique}

As already mentioned, the probability that adjoint MC particles will cross an adjoint detector plane perpendicularly is virtually zero. This probability becomes non-zero when

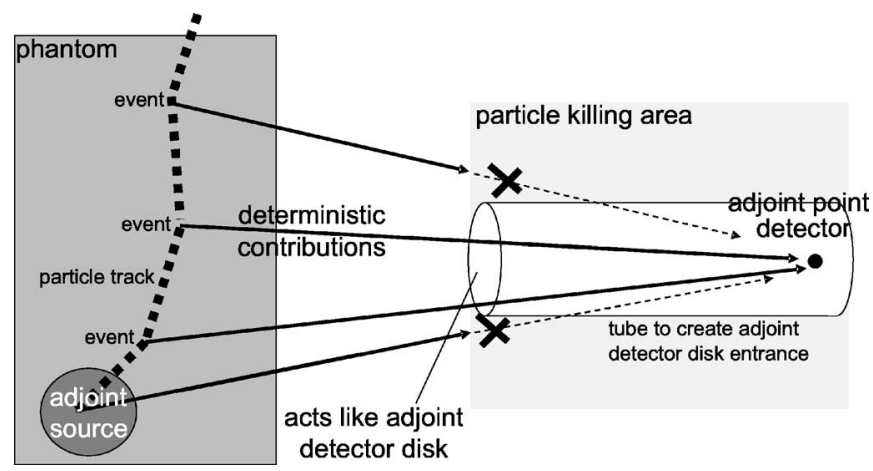

FIG. 1. Adjoint Point Detector Technique: An adjoint point detector far away from the phantom records only deterministic contributions of adjoint particles traveling perpendicular to the tube's entrance. This entrance is shaped like the forward beam exit.

detecting all adjoint particles within a small range of directions. However, it will take a long calculation time to obtain good statistics because many adjoint particles will give no contribution to the adjoint detector. A solution for this problem would be to force the flight direction along the preferred angle just before it crosses the adjoint detector plane. MCNP has a next-event estimator that closely meets this requirement. The technique is called DXTRAN, which in MCNP terminology stands for Deterministic TRANsport. In general, it involves deterministically transporting particles on events to an arbitrary, user-defined sphere in the neighborhood of a detector and then calculating contributions to the detector from these particles. When this user-defined sphere is taken to be infinitely small, DXTRAN is able to obtain many particles in a point of interest that would otherwise be impossible to sample. MCNP tally type 5 is known as the point detector. DXTRAN can be used with both forward and adjoint particles. In this work, the contributions to the point detector are only from adjoint particles. Following an idea from the MCNP-forum, ${ }^{19}$ it is recommended to locate an adjoint point detector very far away from the phantom, such that all the forced contributions are parallel. Using a cylinder, outside of which all adjoint particle histories are killed, effectively creates an adjoint disk detector resembling the beam exit. This technique will be further called the adjoint point detector technique (APDT) and is illustrated in Fig. 1.

In the examples discussed in this work, a distance of $10^{4} \mathrm{~cm}$ between the center of the phantoms and the adjoint point detector is required to have the angle effect disappear and simulate only parallel contributions. This distance is related to the size of the phantom under investigation and the diameter of the tube forming the adjoint disk detector. In fact, the position of the point detector in the cylinder determines the angle of incidence. This means that any divergence of a beam can be simulated by changing the location of the point detector. Due to its forced nature, the relative uncertainty in the APDT decreases rapidly as a function of the number of adjoint particle histories/events whenever a contribution can be made. For the geometries used in the examples described in this paper, there is no reduction in calculation time when using more adjoint point detectors in one 
single MC run when compared with separate runs for each adjoint point detector. In this latter case, the geometry needs to be loaded every time, adding a so-called heating time, but this does not increase the total calculation time.

\section{FET for angular interpolation: The Legendre EXpansion Technique}

The basic idea behind the functional expansion technique (FET) is to describe a detector response by a series expansion using MC samples for estimating the coefficients. Since its earliest reference, ${ }^{12}$ the FET technique has been applied to various radiation problems ${ }^{20-24}$ and its convergence properties analyzed. ${ }^{25}$ Any well-behaving function $f$ can be expressed by

$$
f(x)=\sum_{n=0}^{\infty} d_{n} \psi_{n}(x)
$$

where $x$ is a set of coordinates in the phase space, $\psi_{n}$ are orthonormal basis functions, and $d_{n}$ are coefficients given by the scalar product of

$$
d_{n}=\left(f, \psi_{n}\right) \text {. }
$$

A MC transport simulation can be considered as sampling an implicitly given probability density function (pdf) sample is a set of coordinates in the phase space $(x)$ and the statistical weight of the MC particle $w$. A general response $R$ can usually be described as an integral of the $\wp$ pdf with a chosen detector function $h$. With these definitions, the estimation of the response can be formulated by

$$
R=\int \wp(x) h(x) d x \approx \frac{1}{N} \sum_{i=1}^{N} w_{i} h\left(x_{i}\right)
$$

from $N$ number of $\left(x_{i}, w_{i}\right)$ samples. If the desired response is the value of the implicitly sampled pdf at a coordinate $x_{0}$, the detector function is selected as $h:=\delta\left(x-x_{0}\right)$. Normally, none of the samples fall exactly at $x_{0}$, unless forced by next-event estimators, thus the number of non-zero scores (and, consequently, the convergence rate) is extremely low. This was already mentioned in the Introduction. After applying the general formula for the MC estimator, Eq. (9), together with an estimate for the $d_{n}$ expansion coefficients (truncated at some low number, $M$ ), the pdf can be reconstructed and used to estimate the value of $\wp\left(x_{0}\right)$ by

$$
\begin{aligned}
\wp\left(x_{0}\right) & =\sum_{j=0}^{\infty} d_{j} \psi_{j}\left(x_{0}\right) \\
& =\sum_{j=0}^{\infty} \int \wp(x) \psi_{j}(x) d x \psi_{j}\left(x_{0}\right) \\
& \approx \frac{1}{N} \sum_{i=1}^{N} w_{i} \sum_{j=0}^{M} \psi_{j}\left(x_{i}\right) \psi_{j}\left(x_{0}\right) .
\end{aligned}
$$

In the adjoint particle transport simulation, the estimator of the importance $\left(\phi^{+}\right)$or adjoint function is to be given at a surface, for a certain solid angle $\Omega_{0}$ coinciding with the sur-

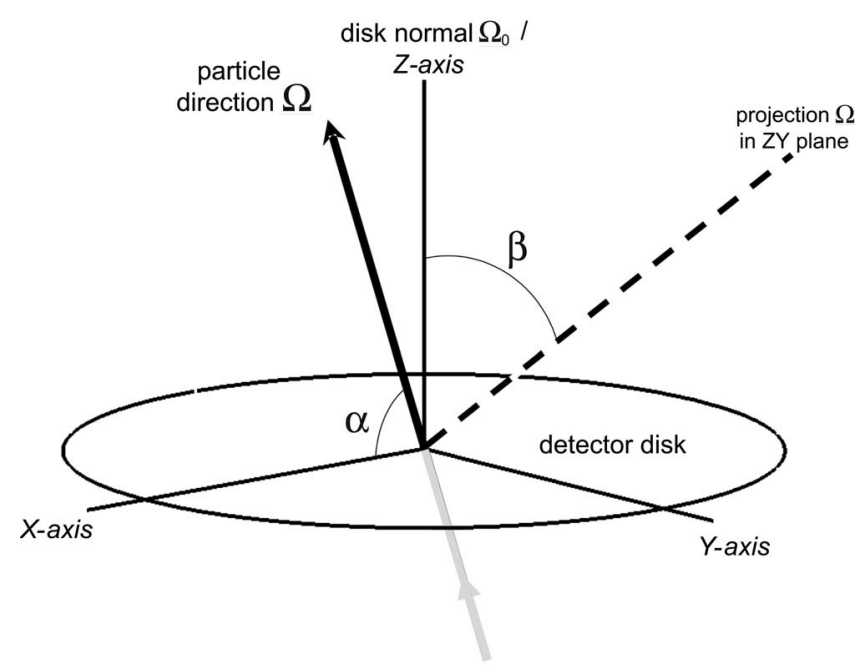

FIG. 2. Adjoint detector disk for Legendre expansion. The direction vector of the crossing adjoint particle is translated into $\alpha$ and $\beta$.

face normal, by applying Eq. (10). As is explained in more detail in the next section, $\Omega_{0}$ is decomposed into two independent angular variables $(\alpha$ and $\beta)$ and, therefore, a twodimensional base function set $\psi_{l, m}(x, y)$ has to be selected. For the base functions the product of two Legendre polynomial $\left(P_{l}\right)$ expansions is used: $\psi_{l, m}(\Omega):=c_{l} c_{m} P_{l}(\cos (\alpha)) P_{m}(\beta)$, where $c_{l, m}$ are the normalization factors. As the Legendre polynomials are orthogonal on $L^{2}(-1,1)$, the angular coordinates should be both transformed to $(-1,1)$. While only Legendre polynomials are applied in this work, it is more appropriate to introduce the term Legendre EXpansion Technique (LEXT) instead of using the general term FET.

\section{E. Parameterization of the LEXT for mono-directional beams}

Adjoint particles traveling parallel to the disk normal are the ones belonging to a mono-directional beam. In Fig. 2, an adjoint detector disk is shown together with its normal and a direction vector $\Omega$ of an adjoint particle crossing the disk. According to the last section, the direction of the normal is defined as $\Omega_{0}$. In the remaining text, whenever "normal" is written the outer normal of the adjoint detector disk is mentioned which is always pointing away from the phantom.

Adjoint particles in a simulation cannot return after crossing the adjoint detector disk. Therefore, the importance is only non-zero in one hemisphere of the solid angle. The Legendre expansion has better pointwise convergence ${ }^{26}$ properties near the middle of $(-1,1)$. Therefore, the parameterization of the hemisphere of the angular coordinates is set to have the disk normal falling in the middle of the range of both $\cos (\alpha)$ and $\beta$. Note that this requirement is something that can be accomplished in several ways. In this work it is chosen that the $X$ - and $Y$-axis of the coordinate system are defined within the disk plane and the $Z$-axis along the normal. $\beta$ is chosen as the angle between the $Z$-axis and the projection of the particle direction in the $Z Y$ plane. Since particles can only pass the disk from one side, $\beta$ is in 
NEUTRONS:

(a)
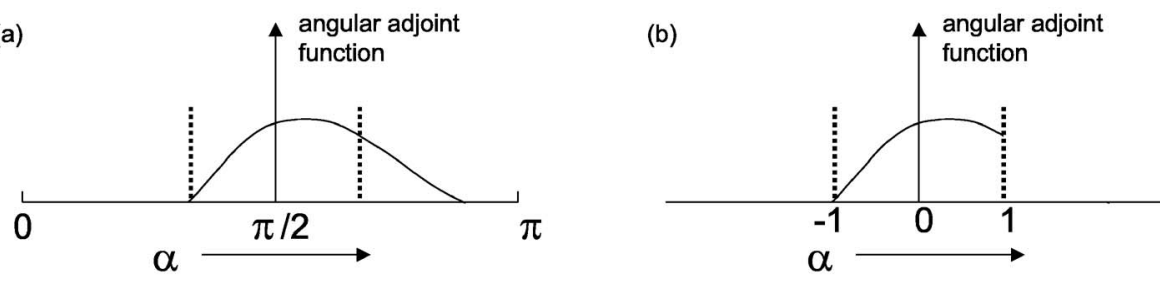

GAMMAS:

(c)

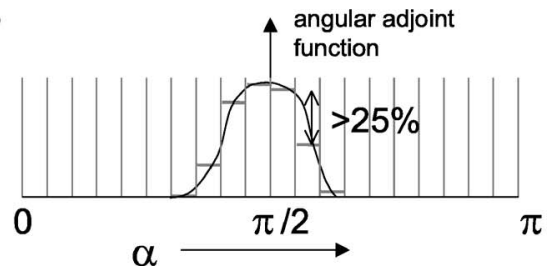

(d)

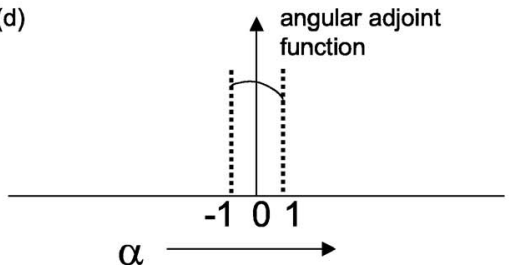

FIG. 3. Neutrons and gammas demand for different truncation methods to exclude zero values or steep gradients in the angular adjoint functions. By truncating the functions, fewer Legendre polynomials are needed for expansion. A typical adjoint angular function for neutrons is shown in the angular domain (a) and Legendre domain (b). A characteristic angular adjoint function for gammas in both domains is shown in (c) and (d). $[-\pi / 2, \pi / 2]$ with the normal direction centered at 0 . Note that $\beta$ is defined negative for negative $Y$ coordinates. Also $\alpha$ is chosen, between the $X$-axis and $\underline{\Omega}$, such that the normal direction is at the center of the interval at $\pi / 2$ in $[0, \pi]$. Both intervals can be translated simply to the Legendre interval of $(-1,1)$. Rewriting Eq. (10) taking into account the Jacobians, omitting all phase space coordinates but the solid angle, and with both $\alpha$ and $\beta$ linearly scaled to $(-1,1)$, gives

$$
\begin{aligned}
\phi^{+}\left(\underline{\Omega}_{0}\right)= & \int \phi^{+}(\underline{\Omega}) \delta\left(\underline{\Omega}_{0}-\underline{\Omega}\right) d \Omega \\
\approx & \frac{1}{N} \sum_{i=1}^{N} w_{i} 4 \sum_{j=0}^{M} \sum_{k=0}^{K} \frac{2 j+1}{2} \frac{2 k+1}{2} \\
& \times P_{j}\left(\cos \alpha_{i}\right) P_{k}\left(\beta_{i}\right) P_{j}\left(\cos \alpha_{0}\right) P_{k}\left(\beta_{0}\right) .
\end{aligned}
$$

Practically, this equation provides a factor that belongs to the direction of the normal for which the initial weight of the adjoint MC particle $\left(w_{i}\right)$ has to be corrected. After this weight correction, the weights can be added and the standard deviation estimated in the regular way. In other words the outcome looks like a normal tally that MCNP would provide.

This interpolation technique works well only if the importance function is a smooth function of the solid angle, and is indeed peaked near the middle of $(-1,1)$. A more sophisticated behavior requires more coefficients for an accurate description. Increasing the polynomial order means higher fluctuations in the correction of the weight. Therefore, while the expansion truncation error decreases, the estimator becomes less stable in the statistical sense. ${ }^{25}$

To reduce the number of Legendre coefficients that otherwise has to be used to describe a function with zero values and/or steep gradients at the edges of the Legendre interval $(-1,1)$, two approaches have been chosen dependent on the particle type. This is explained in the next section.

\section{F. Improvement of the LEXT by reducing the number of coefficients}

The number of Legendre coefficients can be reduced by expanding the angular adjoint function over intervals for $\alpha$ and $\beta$ around the normal direction and truncating the angular domain to exclude discontinuities and to some extent rapidly changing gradients. Possible function discontinuities may arise at the beam endings, as below a certain angle, adjoint particles from the tissue cannot hit the adjoint detector disk. Likewise the exclusion of rapid gradient changes would enhance the convergence at the cost of shrinking the adjoint detector (disk) size, meaning less hits to utilize for the LEXT. After rescaling to the Legendre interval, the normal direction is chosen to be at 0 where good pointwise convergence properties of the Legendre expansion is expected, as already mentioned in Sec. II E.

The angular adjoint functions in human tissue are very different for gammas and neutrons for the particle energies that are involved in this work. The fact that gammas scatter less than neutrons results in steep and narrow shaped angular adjoint functions for gammas whilst broad and flat for neutrons. Therefore, the truncation of $\alpha$ and $\beta$ intervals are performed differently for gammas and neutrons. Two examples of typical angular adjoint functions for neutrons and gammas and the way that intervals of the adjoint functions are truncated are given in Fig. 3. All the procedures and results shown in Fig. 3 are for $\alpha$. For $\beta$, it is the same procedure.

For neutrons the intervals are defined by the zero value of the angular adjoint function closest to the normal direction. For example, in Fig. 3(a), the left zero value is closest to $\alpha$ $=\pi / 2$ (normal direction of the adjoint detector) and defines the symmetrical interval indicated by the dashed lines. This interval is rescaled to $(-1,1)$ in the Legendre domain as illustrated in Fig. 3(b). For gammas the intervals are defined by the steep gradients in the angular adjoint function closest 


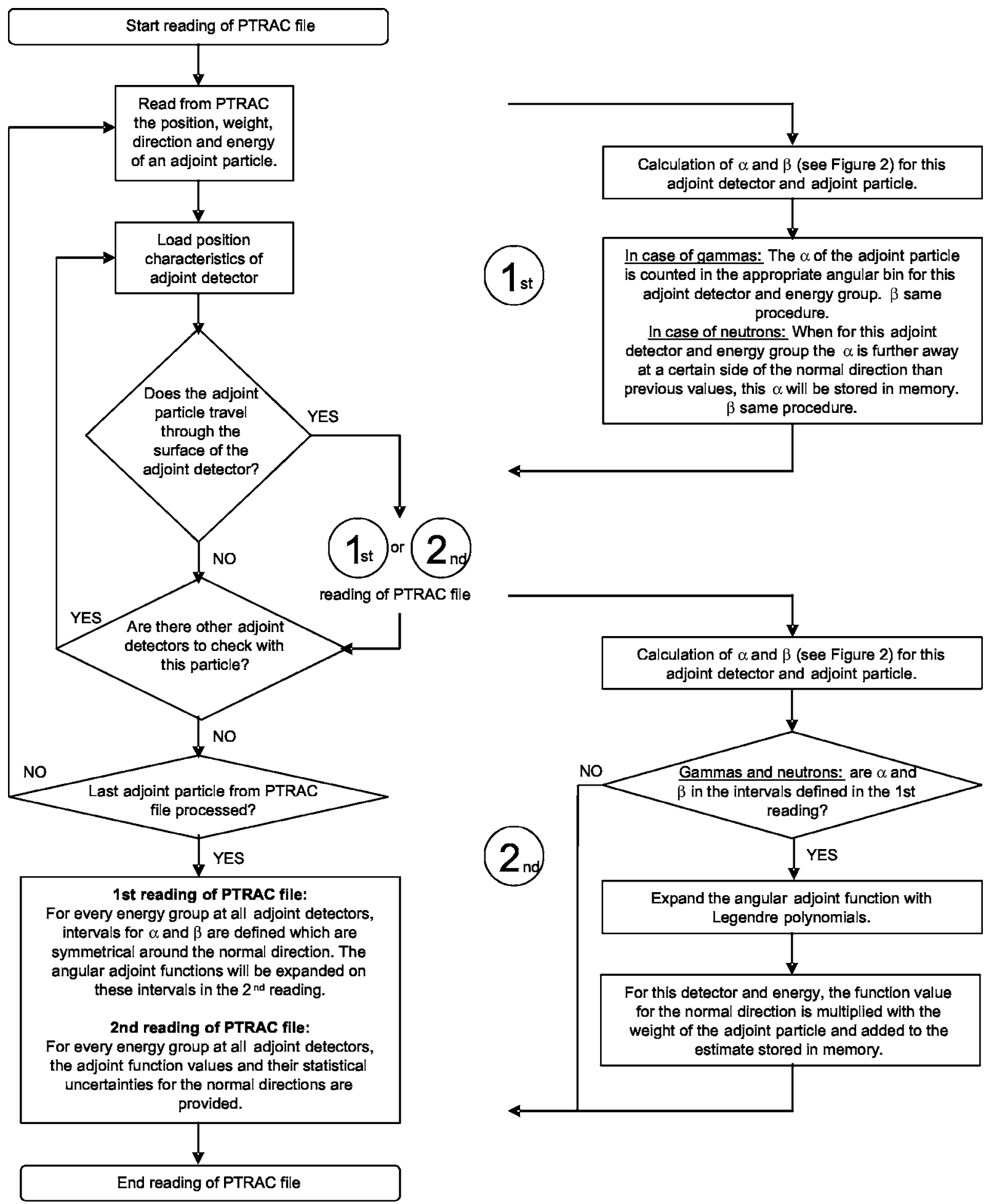

FIG. 4. Schematic overview of all steps involved in the preprocessing for the LEXT. Note that all recorded adjoint particles stored in the PTRAC file have to be read twice.

to the normal direction. The steep gradients can be found by making a course representation of the adjoint function for which the interval for $\alpha$ is divided into 20 angular bins. Figure 3(c) shows an example of an adjoint gamma function and its coarse representation in the form of a histogram. It is chosen in this work to define a steep gradient when the angular adjoint function value of a successive angular bin dif- fers by more than 25\%. Therefore, in Fig. 3(c) the second bin on the right side of the normal direction decreases more than $25 \%$, which causes the function to be expanded for $\alpha$ 's falling within the two bins around the normal [see Fig. 3(d)].

Consequently, for the techniques described above for gammas and neutrons it has to be considered that by truncating the intervals for $\alpha$ and $\beta$, adjoint particle information 
outside the intervals is not used. This could result in an estimate from the LEXT which is poor while based on a few samples. To prevent this situation, it is chosen in this work to check if at least $50 \%$ of all samples for every energy group and adjoint detector are used. However, it seems that the steep and narrow-shaped adjoint gamma functions only sometimes encounter this problem. In these cases, successive angular bins are added until $50 \%$ of the samples are included.

Although, the techniques and assumptions presented here to improve the LEXT work fine for the examples discussed in Secs III and IV, it is worthwhile studying this aspect in more detail.

\section{G. Adjoint particle recording and pre-processing for the LEXT}

The first step in the LEXT to determine the contribution of a mono-directional beam to a specified region in a phantom is to perform an adjoint MC calculation without defining any adjoint detector. The adjoint source particles start isotropically in a cell which can be a tumor or OAR, interact and gain energy by traveling through the phantom. Adjoint particles that leave the phantom will travel through the surrounding air and are recorded when they cross a sphere surrounding the phantom. In this study, the adjoint particles are recorded when they pass a sphere with a radius of $50 \mathrm{~cm}$. The computer output file in which the position, direction, weight, and energy of the adjoint particles are recorded is called a PTRAC file. This MCNP terminology comes from Particle TRACk output and allows the user to gather each individual particle event. Normally, individual particle events are lost, but are recorded as a contribution to the estimate and its statistical uncertainty of a certain MCNP detector. In order to keep the size of the PTRAC file within limits, the user can define filters. In this work only adjoint particles crossing the mentioned sphere around the phantom pass the filter and are recorded. This sphere will be further called the PTRAC sphere.

After the adjoint MC calculation is finished, custom made Fortran routines are used for preprocessing. To obtain the results, the PTRAC file, containing all recorded adjoint particles, has to be read twice. The first reading is necessary to obtain for all adjoint detectors and all energy groups, a part of the adjoint angular function around the normal direction which has no steep gradients or zero values as explained in Sec. II F. The second reading of the PTRAC file is performed to expand the angular adjoint function with Legendre polynomials and to provide an estimate for the normal directions of all adjoint detectors for each energy group. A detailed overview of all steps involved in the preprocessing for the LEXT is given in Fig. 4. Apart from the PTRAC file, the preprocessing needs a list with the spatial information of all adjoint detectors the user is interested in.

All calculations in this study, done with MCNP version $4 \mathrm{C} 2$ as well as with the postprocessing codes written in Fortran, are performed on a Pentium IV personal computer with a $3 \mathrm{GHz}$ processor and $512 \mathrm{Mb}$ of memory, in a Windows

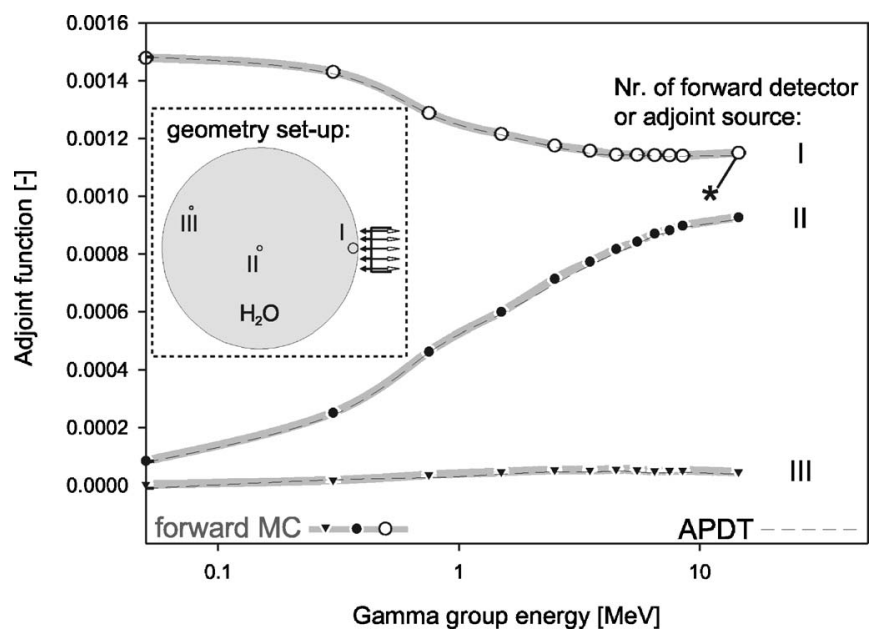

FIG. 5. Contributions of source gammas, of 12 different energy groups which leave a $10 \mathrm{~cm}$ diam source disk perpendicularly, to three different cells in a light water sphere. These so-called adjoint functions are obtained with APDT and forward MC which give identical results.

XP command shell. This information is given in order to interpret the absolute times given in the results section. All the calculation times given include the loading time of the geometries (heating time).

\section{EXAMPLE 1: WATER SPHERE IRRADIATED WITH GAMMAS TO SHOW VALIDITY OF THE APDT AND CONVERGENCE OF THE LEXT}

The purpose of this first example is to show that the APDT and LEXT generate the same results as can be obtained with forward MC. This will be done by comparing the adjoint functions calculated with the APDT and forward MC. The way to calculate adjoint functions with forward MC is described in the last paragraph of Sec. II B. Furthermore, this example is used to illustrate the physical significance of adjoint functions. Secondly, the APDT and LEXT results are compared, with the focus on how the LEXT result converges towards the APDT when using the correct number of Legendre coefficients.

\section{A. Set-up}

For this example, a spherical light water phantom is chosen which is irradiated with mono-directional gammas coming from a $10 \mathrm{~cm}$ diam disk shaped source. A cross section of the geometry set-up is shown in the inset in Fig. 5. The water sphere has a diameter of $40 \mathrm{~cm}$ and contains three spherical cells with diameters of $2.0,1.0$, and $0.5 \mathrm{~cm}$ which are filled with water and are positioned at different distances from the source. These cells are labeled I, II, and III, respectively, in the inset in Fig. 5. The goal is to obtain the energy-dependent adjoint functions in these three cells. In the forward MC set-up the three cells are forward detectors and the forward source is at the right side. The direction of the forward source particles is towards the light water phantom as indicated with the arrows. The "open" arrows in the opposite direction represent the adjoint particles of the adjoint calcu- 
lation for the APDT. These adjoint particles travel parallel through the $10 \mathrm{~cm}$ disk towards the point detector which is $10^{4} \mathrm{~cm}$ away and not shown. For both the APDT and LEXT, the cells I to III become the adjoint sources in which the adjoint particle tracks start in isotropical directions. For the three MC methods, the gamma multigroup cross sections and energy group structure from the MENDF ${ }^{27}$ library are applied. The forward and adjoint sources have a uniform energy spectrum over 12 energy groups between the limits of $0.01 \mathrm{MeV}$ up to $20.0 \mathrm{MeV}$. The detector response functions for the forward and adjoint methods are similar and also taken uniform over the 12 energy groups. In this example, the group results are presented at the central energies of the 12 groups. In MCNP the forward detectors are modeled as volume tallies of the F4 type and the point detector in the APDT is of type F5. For the LEXT there is no tally set, as the adjoint particles are recorded in the PTRAC file when passing the PTRAC sphere.

\section{B. Results: Comparison between the APDT and forward MC}

The graphs in Fig. 5 show six adjoint functions which are obtained in the spherical cells I, II, and III with forward MC and the APDT. These adjoint functions belong to the direction of the normal of the adjoint detector disk. It can be clearly seen that the results of both methods are very similar. To obtain similarly averaged statistical uncertainties in the 95\% confidence interval, which for cells I to III is $0.1 \%$, $0.3 \%$, and $2 \%$, respectively, the forward MC takes $30.8 \mathrm{~min}$ against $2.2 \mathrm{~min}$ for the APDT. It is clear that adjoint MC performed with the APDT has a clear advantage for these kinds of set-ups with relatively small forward detectors compared with the size of the forward sources.

The adjoint functions in Fig. 5 indicate the number of source gammas of a certain energy group leaving the $10 \mathrm{~cm}$ diam disk perpendicularly that contribute to the cells I, II, and III. For example, see the function value indicated with an asterisk in Fig. 5. At this point, per source gamma leaving the $10 \mathrm{~cm}$ diam disk-shaped source, around 0.0012 source gammas with an energy between 9 and $20 \mathrm{MeV}$ (the 12th energy group) contribute to cell I. In other words: out of every 833 source gammas, there is one source gamma having an energy between 9 and $20 \mathrm{MeV}$ which contributes to cell I.

Concerning the relative behavior and positioning of the three curves it is obvious that adjoint function I is the highest since it is positioned closest to the source. Compared with the other cells, many source gammas of all energy groups can contribute easily to cell I because they are not affected by the water thickness (depth). Curve I is higher for low gamma source energies, as these gammas have a higher probability to scatter in the first few centimeters in the water and be directed towards cell I. For the smaller cell II, located at a further distance from the source, only the higher source gamma energies contribute. Most of the lower energy gammas are already stopped or scattered in the water. The smallest cell III is furthest away from the source and positioned

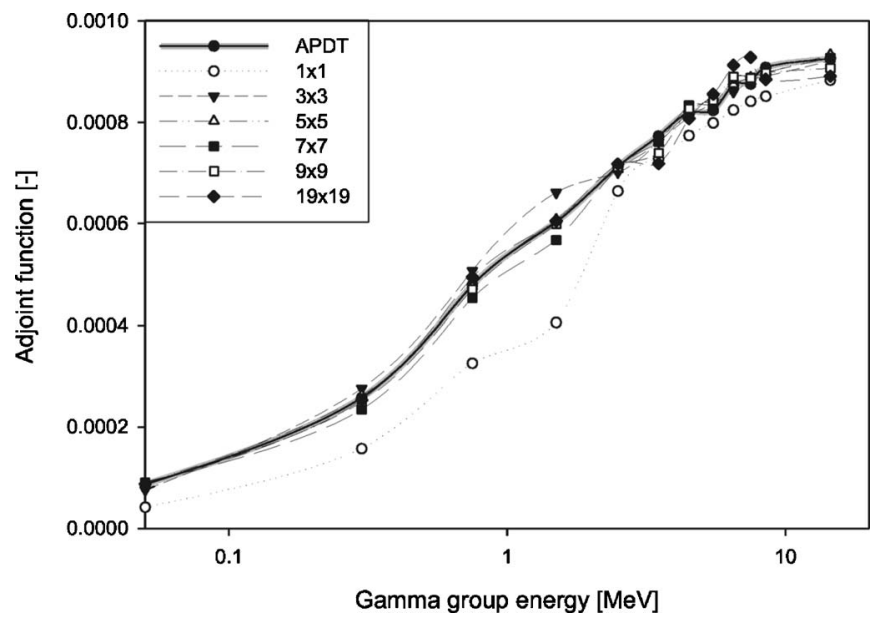

FIG. 6. The adjoint functions in cell II obtained with the LEXT for different numbers of Legendre coefficients used for $\alpha$ and $\beta$. The LEXT converges towards the APDT, which is the reference when using five coefficients or more for $\alpha$ and $\beta$.

outside the direct gamma beam. Only a very small number of the source gammas having the highest energies will scatter such that they can contribute to this cell.

\section{Results: Convergence of the LEXT}

A further comparison is made between the adjoint functions obtained with the LEXT and APDT for cell II. For this purpose, an adjoint MCNP calculation for the LEXT is started with $3 \times 10^{8}$ adjoint source gammas starting in cell II. This simulation and writing of the $35 \mathrm{~Gb}$ PTRAC file takes 131 min of CPU time. In Fig. 6, the gray line represents the adjoint function obtained with the APDT which is now used as the reference. To obtain the adjoint function with the LEXT, the correct number of Legendre coefficients has to be determined. In this example, the $\mathrm{M}$ and $\mathrm{K}$ for $\alpha$ and $\beta$ in Eq. (11) are both taken as 1, 3, 5, 7, 9, and 19 coefficients. For these numbers of coefficients, the adjoint functions are shown in Fig. 6. Starting with one Legendre coefficient for $\alpha$ and one for $\beta$ (written as $1 \times 1$ ), it can be seen that the LEXT result does not agree with the APDT. The curve rises for 3 $\times 3$ coefficients and then from $5 \times 5$ to $9 \times 9$, the adjoint function obtained with the LEXT has for this configuration converged. Making the same sets of curves for the other positions of the cells, i.e., cells I and III, it is observed that all adjoint functions have $\mathrm{a}<2 \%$ average difference with the corresponding APDT result, when using $7 \times 7$ Legendre coefficients in the LEXT. Also further experiments in which the orientation and diameter of the adjoint detector is changed indicate that using $7 \times 7$ coefficients continues to generate the same adjoint functions as the APDT and forward MC.

When even more coefficients are used, the results start to oscillate (see, for example, $19 \times 19$ in Fig. 6) and also the relative errors increase. The relative errors $(95 \%$ confidence interval) of the above results are given in Fig. 7. The more coefficients are used, the higher the relative error becomes. Consequently, the optimal number of coefficients has to be 


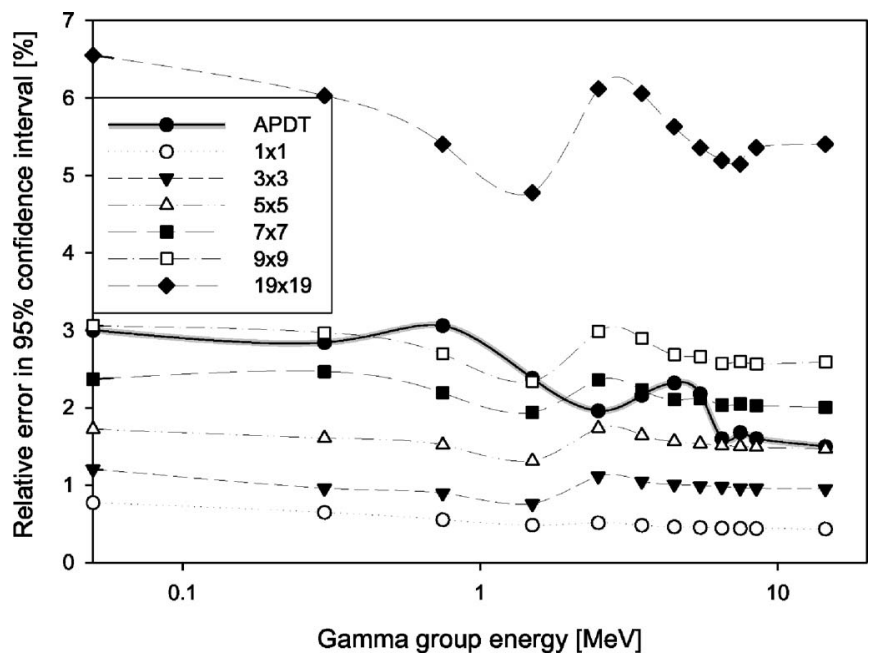

FIG. 7. The relative errors in the adjoint functions for the LEXT and APDT. The errors of the LEXT increase with increasing number of Legendre coefficients used for $\alpha$ and $\beta$.

chosen such that the result converges with the minimum relative error. This approach is further discussed in Sec. V A.

For the comparison with the LEXT, the APDT result is obtained with $3.5 \times 10^{5}$ adjoint source gammas such that the average relative error is the same as for the LEXT with 7 $\times 7$ coefficients. In this case, the calculation for one adjoint detector disk with the APDT takes $8 \mathrm{~s}$ and proves that the LEXT cannot compete regarding the calculation time. The LEXT will start to profit when more adjoint detectors are involved because the already-written PTRAC file contains all the information around the phantom. For now it is concluded that the two adjoint MC methods give identical results as forward MC and that the LEXT converges for a certain geometry when applying a tuned number of coefficients.

\section{EXAMPLE 2: PHANTOM HEAD IRRADIATED WITH NEUTRON AND GAMMA BEAMS}

This example illustrates how the APDT and LEXT perform in comparison with forward $\mathrm{MC}$ in a real radiotherapy BNCT scenario. The calculation times of the three methods to obtain the total detector responses in the tumors and OAR are compared when irradiating a human head. This is performed with neutrons and gammas. The way to compare the three methods is discussed in Sec. IV C. In the results sections, the calculation times are presented and a preliminary result is shown indicating the optimal neutron beams around the patient's head. After all, the goal of these adjoint methods is to increase the number of beam scenarios that can be calculated in a certain amount of time.

\section{A. Monte Carlo set-up}

In this example, the boundary source $\phi^{b}$ [see Eq. (5)], represents the treatment beam as used in the BNCT trials at the high flux reactor in Petten, The Netherlands. The beam can be regarded as mono-directional (angular divergence $<2^{\circ}$ ) and consists mainly of epithermal neutrons around
$10 \mathrm{keV}$. The relative error in the source spectrum of the beam is taken as zero. In the forward MC calculations, the detector response function $\Sigma_{d}$ in Eq. (5), should have an energy spectrum in accordance with the absorption crosssections of ${ }^{10} \mathrm{~B}, \mathrm{H}$, and $\mathrm{N}$. These cross-sections have in common that at thermal energies, the probability for a reaction is orders of magnitude higher than at high neutron energies. As such, in BNCT, determining the total detector response due to the flux of thermal neutrons with energies $<0.5 \mathrm{eV}$ provides a good impression of the main (thermal neutron related) dose components. Therefore, the (forward) detector response function $\Sigma_{d}$ in tumors and OAR is taken uniform up to $0.5 \mathrm{eV}$. The optimization of a BNCT treatment plan from the neutronics point of view is all about getting a high thermal neutron flux in the tumors and a low thermal neutron flux in the OAR.

For the forward and adjoint MCNP neutron calculations in this example, a new 172-group library in the XMAS energy structure based on JEF-2.2, ${ }^{28}$ is used. The library is made for a temperature of $37{ }^{\circ} \mathrm{C}$ and the $S(\alpha, \beta)$ thermal scattering treatment for hydrogen is taken into account. In the APDT and LEXT, the adjoint detector response function is similar to the (forward) boundary source function $\phi^{b}$ which is the energy spectrum of the treatment beam in Petten in the 172 groups representation. Vice versa, the adjoint source function is similar to the (forward) detector response function $\Sigma_{d}$. In this example, for the three methods, the gamma settings are chosen similar to the ones used in Example 1. This means that the total detector response due to the flux of gammas in tumors and OAR is calculated for a uniform gamma source with energy limits between $0.01 \mathrm{MeV}$ up to $20.0 \mathrm{MeV}$. In this section, "total detector response due to a flux" is sometimes shortened by mentioning only "flux."

\section{B. Geometry set-up}

From 125 CT images of a patient's head, a voxelized MCNP geometry is made using the program Scan2MCNP. ${ }^{29}$ The head has a width of $17.1 \mathrm{~cm}$, a height of $20.8 \mathrm{~cm}$, and the distance from the tip of the nose to the back of the head measures $22.2 \mathrm{~cm}$. According to the gray intensity of a pixel in the CT image, a threshold can be set and a tissue material assigned. The outer layer of the head is modeled as soft tissue representing a mixture of skin, muscle, and fat. The phantom head consists of 47520 voxels which contain only or mixtures of air, brain tissue, soft tissue, and cranium. These tissue compositions and densities are taken from ICRU46. ${ }^{30}$ In Fig. 8, a 3D plot (made with Sabrina ${ }^{31,32}$ ) of the head is shown with all tissue materials set semitransparent.

Ten spherical tumor lesions, which is not unusual for patients with melanoma metastases in the brain, are defined (dark colored), together with the ten OAR (light colored), as defined in the BNCT protocol ${ }^{33}$ used in Petten. The diameters of the tumors are all $1 \mathrm{~cm}$. Similar to the tumors, all OAR are modeled as spheres and represent the eyes, inner ears, pituitary gland, parotid glands, region of the thalamic vessels, and chiasma opticum. The diameters of the OAR 


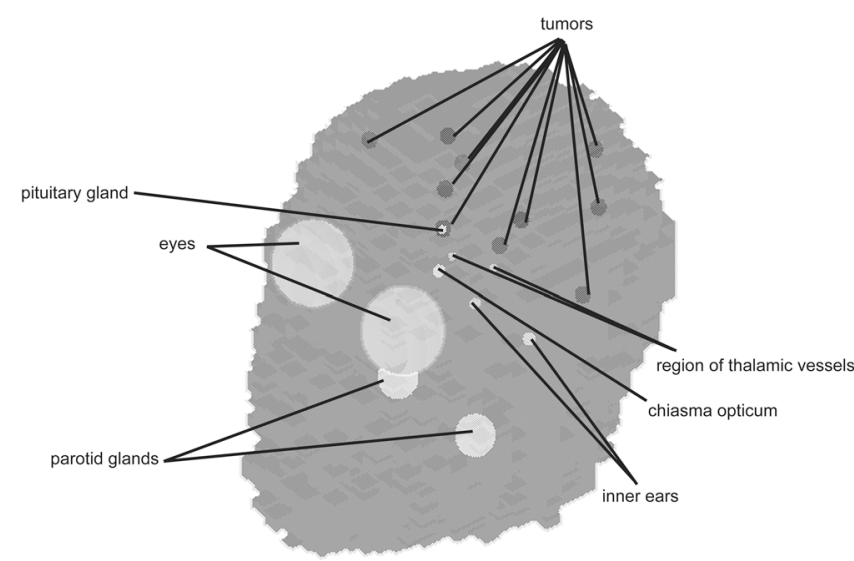

FIG. 8. Head phantom with all tissues set semi-transparent, the ten OAR are light and the ten tumors dark colored.

range from 4 to $0.5 \mathrm{~cm}$ of which the last merely indicates a spot. All these 20 tumors/OAR are the adjoint sources in the adjoint $\mathrm{MC}$ techniques and forward detectors in the forward MC calculations.

In this study, for the LEXT, there are 60 adjoint detector disk center points which are closely packed ${ }^{34}$ and distributed spherically around the head. The disk center points are $20 \mathrm{~cm}$ away from the center of the phantom that is also the center of the PTRAC sphere and are described in azimuthal and polar angles, as shown in Figs. 9(a) and 9(b). The initial orientations of the 60 adjoint detector disks are defined as having the inner normal pointing at the center of the phantom. This is illustrated for one disk in Fig. 9(b). In order to investigate more beam directions, at each of the 60 center points, 16 extra detector disks with different orientations are added.

As shown in Fig. 9(c), eight disks have normals making angles of $10^{\circ}$ with the initial disk normal; these normals are numbered 2-9. Another eight normals make an angle of $20^{\circ}$ with the initial normal [numbered 10-17 in Fig. 9(c)]. All these extra adjoint detectors are generated and numbered using the same routine for each of the 60 points around the head. This infers 17 disk orientations for each of the 60 positions to give 1020 adjoint detectors at which the adjoint function is determined. This implies that the effect of 1020 "Petten" neutron/gamma beams can be investigated. In the forward MC calculations these 1020 disks are the forward sources (treatment beam exits). In the APDT these 1020 disks act like adjoint detector disks as illustrated in Fig. 1. In order to observe the influence of the diameter of the treatment beam on the three methods, all 1020 beams can have beam exit diameters of 5, 10, and $15 \mathrm{~cm}$. In MCNP, for both neutrons and gammas, the forward detectors are modeled as volume tallies of the F4 type and the point detector in the APDT is of type F5.

\section{Strategy for comparing the calculation times of the three methods}

Comparison of the calculation times of the forward MC calculations, the APDT and the LEXT, is based on a comparison of the relative errors obtained in the tumors and OAR, averaged over a certain number of beams. This relative error belongs to the total detector response as defined in Sec. II B. The LEXT is calculated for all 1020 beams with

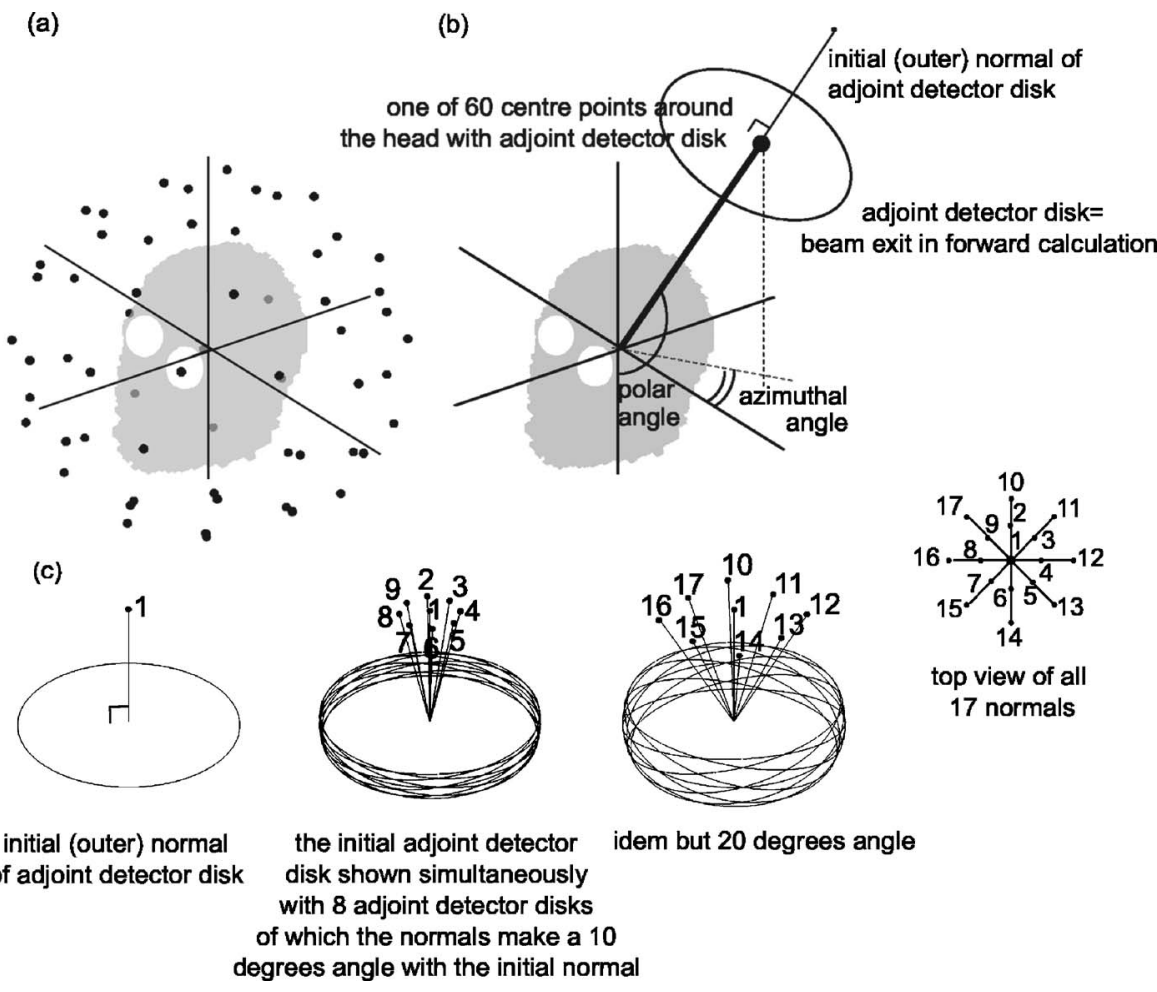

FIG. 9. (a) The 60 center points of the adjoint detector disks around the head. (b) These points are described with polar and azimuthal angles. (c) Orientation of the 17 adjoint detector disks and their normals, which is similarly the case at each of the 60 center points. In total there are $60 \times 17$ $=1020$ beams simulated which can have diameters of 5,10 , and $15 \mathrm{~cm}$. 


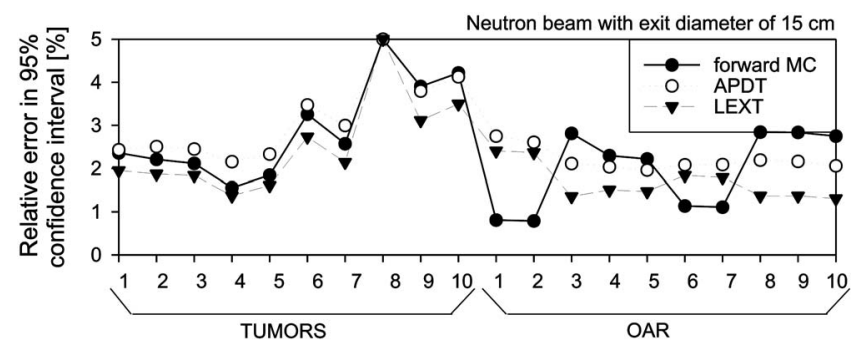

FIG. 10. The relative errors in the total detector responses due to the flux of thermal neutrons $(<0.5 \mathrm{eV})$ in all tumors and OAR. The errors for the three methods are normalized to a maximum of $5 \%$ and averaged over 255 neutron beams after which the calculation times of the methods can be adapted and compared.

three different diameters. As presented later in Sec. IV D, to calculate all 1020 beams with three different diameters for all methods is very time consuming. For this purpose, for each beam diameter, the APDT and forward MC are only calculated for 255 beams out of the 1020. These beams are selected proportionally around the head and checked if the standard deviations of the relative errors converge for these 255 beams.

Knowing that the relative error in a MC calculation relates to the square root of the number of histories, it can be calculated how much longer or shorter the MC runs should be in order get a certain relative error. The pre-processing time of the LEXT scales linearly with the number of histories to be processed. It is chosen to set the maximum averaged relative error in the tumors and OAR to 5\% (95\% confidence interval). The comparison of the calculation times is based on the adapted time it takes for the LEXT to obtain the results for 1020 beams. The averaged adapted calculation times of the 255 APDT and forward calculations are multiplied by four.

\section{Results: Calculation times of the three methods}

For the case of the neutrons, for the LEXT, a $20 \mathrm{~Gb}$ PTRAC file is written for the tumors and OAR. For the gammas the file has a size of $130 \mathrm{~Gb}$. More gamma samples are needed to obtain the "steep" and "narrow" angular adjoint functions with the required statistics. Since gammas are tracked faster, it takes only 1.5 times longer to obtain the $130 \mathrm{~Gb}$ file in comparison with the neutron PTRAC file. The time to obtain the optimum number of Legendre coefficients (in this example $5 \times 5$ for the neutrons and $7 \times 7$ for the gammas) is excluded from the total time for reasons discussed in Sec. V A.

Figure 10 gives an example of the averaged relative errors over 255 neutron beams with a diameter of $15 \mathrm{~cm}$, after adapting the calculation times of the three methods in order to get the same maximum relative error.

The relative errors of the forward method are relatively small for OAR 1,2,6, and 7, which are the relatively large eyes and parotid glands. This is due to the fact that forward particles generally score better in large detectors. Overall, Fig. 10 shows that the relative errors of the three methods are in the same range and deliver equal quality in the results. The
TABLE I. The total calculation times for the three methods for neutrons. All calculations are performed in a Windows XP command shell on a personal computer with a Pentium IV $3 \mathrm{GHz}$ processor and $512 \mathrm{Mb}$ of memory.

\begin{tabular}{cccc}
\hline \hline & \multicolumn{3}{c}{$\begin{array}{c}\text { Time to calculate 1020 beams } \\
\text { normalized to forward MC }\end{array}$} \\
\cline { 2 - 4 } $\begin{array}{c}\text { Diameter of adjoint detector/ } \\
\text { beam exit }(\mathrm{cm})\end{array}$ & Forward MC & APDT & LEXT \\
\hline 5 & $1.00(=140$ days $)$ & 27.40 & 1.52 \\
10 & $1.00(=113$ days $)$ & 18.42 & 0.15 \\
15 & $1.00(=88$ days $)$ & 12.94 & 0.05 \\
\hline
\end{tabular}

relative error graphs for the other beam diameters and gammas are not shown here, but are similar to Fig. 10.

Table I contains the calculation times for the three methods, for 1020 beams, to obtain the total detector responses of the thermal neutron flux with an equal maximum averaged relative error of 5\% (in $95 \%$ confidence interval), in one of the ten tumors and ten OAR. The calculation times are given for the three diameters. Regarding Table I, due to a great number of neutron events, which are all tracked but cannot score to the point detector, the APDT takes 27 to 13 times longer when compared with the forward calculations; these calculations would take years. For 1020 beams and 20 tumors/OAR, the calculation of the contributions of beams with a diameter of $5 \mathrm{~cm}$ is best performed with forward calculations. Beams with diameters of 10 and $15 \mathrm{~cm}$ can be better calculated with the LEXT: more than 6 and 20 times faster, respectively. Obviously, the advantage of the LEXT becomes more apparent as the beam diameter becomes larger. In spite of the results obtained in the first example, it is demonstrated here that the LEXT becomes faster than the APDT whenever more beam positions are calculated.

In Table II, the gamma results for the head phantom are presented. As there are far less gamma events in the phantom, the APDT improves greatly from that compared with the neutron case. For gamma beams with diameters of 10 and $15 \mathrm{~cm}$, the APDT and LEXT are roughly 2 and 3 times faster, respectively, than forward MC. Note that the APDT will become advantageous over the LEXT when less than 1020 beams are required. In this case, also the preprocessing of the LEXT will take less time but still, to get similar statistics, the same PTRAC file has to be obtained which is relatively time consuming.

TABLE II. The total calculation times for the three methods for gammas. All calculations are performed in a Windows XP command shell on a personal computer with a Pentium IV $3 \mathrm{GHz}$ processor and $512 \mathrm{Mb}$ of memory.

\begin{tabular}{cccc}
\hline \hline & \multicolumn{3}{c}{$\begin{array}{c}\text { Time to calculate } 1020 \text { beams } \\
\text { normalized to forward MC }\end{array}$} \\
\cline { 2 - 4 } $\begin{array}{c}\text { Diameter of adjoint detector/ } \\
\text { beam exit }[\mathrm{cm}]\end{array}$ & Forward MC & APDT & LEXT \\
\hline 5 & $1.00(=53$ days $)$ & 1.57 & 1.47 \\
10 & $1.00(=42$ days $)$ & 0.52 & 0.55 \\
15 & $1.00(=27$ days $)$ & 0.33 & 0.30 \\
\hline \hline
\end{tabular}




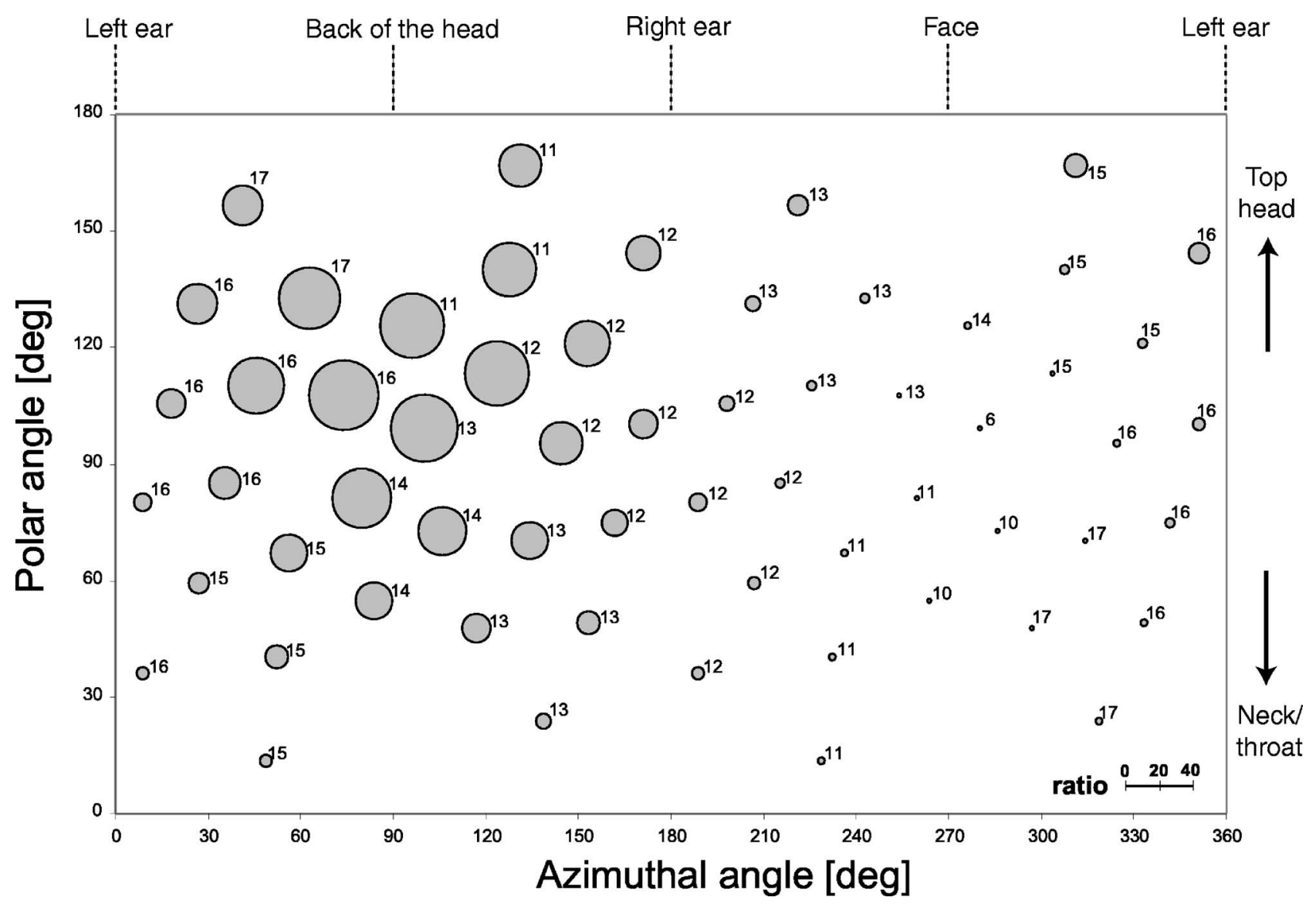

FIG. 11. An example outcome for treatment planning with the $15 \mathrm{~cm}$ neutron beam in Petten: for each of the 60 beam locations around the head, the maximum (=optimal) ratio of thermal neutrons in the tumors to thermal neutrons in the OAR is calculated out of 17 beam orientations. The larger the gray bubble the better is the ratio.

The gamma beams with the smallest exit of $5 \mathrm{~cm}$ diameter are again most quickly obtained with 1020 forward MC calculations.

\section{E. Results: Optimum neutron beams for irradiation}

As an example, the result of what are termed the best "Petten" neutron beams (diameter $15 \mathrm{~cm}$ ) out of 17 orientations at every of the 60 positions are shown in Fig. 11. These in total 1020 neutron beams are calculated with the LEXT, which is 20 times faster than forward MC. The larger the gray bubble in Fig. 11, the larger is the maximum ratio of average thermal neutron flux in all tumors to the average thermal neutron flux in all OAR. In other words, large gray bubbles represent a high dose in the tumor and a low dose in the OAR. All relative errors of the ratios presented in Fig. 11 are $<5 \%$ in the $95 \%$ confidence interval. The numbers written next to each gray bubble correspond to the optimum orientation. The result is checked by the 255 forward calculations and the differences are according to the statistical uncertainties.

When irradiating at some positions at the back of the head, where the largest bubbles are located in Fig. 11, the average thermal neutron flux will be around 35 times higher in the tumors than in the OAR. The small bubble indicated with " 6 " in the facial area has a ratio of 0.3 . This means that the average thermal neutron flux in the tumors is more than 3 times less than in the OAR. Nevertheless, this is the optimal beam out of 17 orientations from this position. To avoid the situation in which the average flux in the tumors is small and in the OAR is nearly zero, which would give anyway a high ratio, the optimum beam orientation is chosen such that $75 \%$ or more of the maximum possible thermal neutron flux in the tumors is always provided.

For the optimization presented here, to determine the best beams it is chosen to perform this by using the thermal neutron flux averaged over all tumors and the thermal neutron flux averaged over all OAR. It needs to be investigated whether this approach gives the same (optimum) treatment plan when compared to the case when the contribution of every beam to each tumor and each OAR is optimized using an optimization scheme. The answer to this question is a subject for future work. Nevertheless, optimization can be studied more quickly with the LEXT and APDT approaches.

\section{DISCUSSION}

In this paper, two adjoint MC techniques are introduced, with an explanation on how perpendicular contributions to an adjoint detector plane can be calculated. Above all, it is the aim of this work to prove that these adjoint techniques work and that they decrease the calculation time per beam in 
MC based radiotherapy treatment planning when compared with forward MC calculations. In this way more beams can be calculated and, as a result, the treatment plans for monodirectional beams can be improved. In realistic scenarios, the outcome for both APDT and LEXT is positive and proven in the examples. For the LEXT, some choices and assumptions are made which need some further attention. This is discussed in the next two sections.

\section{A. LEXT coefficient training}

In the presented calculation times of the LEXT, the times to find the number of Legendre coefficients for which the results converge are excluded. It seems that this number of Legendre coefficients depends on the irradiated geometry and type of particle it is irradiated with. The size of the adjoint detector is of no influence. It is to be expected that when irradiating similar phantoms, such as head phantoms, under similar conditions, this "training for coefficients" needs to be done only once. Nevertheless, although this statement is checked for the geometries presented in Examples 1 and 2, in which the adjoint sources are at various locations, a further investigation is necessary. In this respect, Griesheimer et al..$^{25}$ describe a first approach for a coefficient filtering technique that automates the selection of the optimum number of coefficients. This should be tested and implemented whenever it works properly for the radiotherapy application presented here.

\section{B. Improvements}

A way to improve the LEXT is to investigate other function expansion bases such as spherical harmonics or Fourier or any other function that can describe the angular adjoint function better by definition. The observed difference in the shape of the adjoint functions between neutrons and gammas suggests that the selection of a function expansion bases depending on the particle type, will improve the outcome. Such an investigation, in conjunction with investigating how to select the best samples for the estimates, as described in Sec. II F, is subject to further research. As well as improvements, work can also be done to adapt the LEXT such that it can be used for more divergent beams. The BNCT neutron beam in Petten has a $2^{\circ}$ angular divergence, which gives insignificant differences when simulated by a mono-directional neutron beam. Actually, the diameter of the beam should be adapted for the distance between the beam opening and the irradiated body. Details like this have not been applied, as it was chosen to demonstrate the methods by using Example 2, which is realistic but simplified. Note that in this respect, in Example 2, the distance of the detector disks is chosen as fixed with respect to the center of the phantom instead of the distance to the skin.

\section{CONCLUSION}

A certain total detector response of the flux, dose or reaction rate somewhere in a phantom, due to a known source, can be obtained with forward MC calculations and adjoint
MC calculations. The product of the adjoint function and the source function integrated over all phase-space variables provides this total (forward) detector response. In general, whether an adjoint MC calculation is advantageous over a forward MC calculation, in terms of speed, depends on the number of sources, detectors, and their respective sizes. For example, the results for many large forward sources, and/or a few small forward detectors (=adjoint sources), can be best calculated with adjoint MC. Apart from the evident cases, one can imagine that when the number of sources, detectors, and their sizes vary over certain ranges, it is not straightforward to know whether forward MC is preferable over adjoint $\mathrm{MC}$ or vice versa. It will need experience to determine this and the second example presented in this work can, therefore, in this context only be regarded as an example for radiotherapy treatment planning of a human head. This example shows that 20 adjoint sources (tumors/OAR) can still be regarded as a "few," whenever 1020 adjoint detectors (beams) are simulated.

Up to now, in $\mathrm{MC}$ based radiotherapy treatment planning, adjoint MC calculations are impossible when the treatment beams are mono-directional. This is in despite of the fact that the circumstances are in favor of the adjoint as described above. The LEXT and APDT are two techniques providing an estimate for the value of the angular adjoint function at the direction of the adjoint detector's normal. A disadvantage of the LEXT is that for different types of phantom geometries, the number of Legendre coefficients has to be determined for which the result is converged. The APDT is disadvantageous when there are many events which are not contributing to the result, such as with neutrons in light water.

From the BNCT example of a human head phantom with 20 tumors/OAR and 1020 different positions of a monodirectional neutron or gamma beam, it seems that small beams (exit diameters of $5 \mathrm{~cm}$ ) are calculated most quickly with normal forward MC calculations. This result is valid for gammas as well as neutrons. For the larger beam exits $(10$ and $15 \mathrm{~cm}$ ), for neutrons alone, the adjoint MC calculations based on the LEXT are 6 and 20 times faster, respectively, than the forward MC. For the same beam exit diameters, both the LEXT and APDT are 2 and 3 times faster than the forward MC for the gamma beams. Nevertheless, the APDT will become advantageous whenever fewer beam positions have to be calculated, e.g., beams directed to the face in the head phantom example may be unnecessary, i.e., are unacceptable.

To conclude, prior to this study adjoint MC calculations always showed great potential for MC based radiotherapy treatment planning but suffered when mono-directional treatment beams are considered. From the work presented here, this problem has now been solved and the optimization of treatment plans and/or studying its mechanisms becomes possible.

\section{ACKNOWLEDGMENT}

This work has been supported financially by the Joint Research Centre of the European Commission. 
${ }^{a)}$ Electronic mail: victor.nievaart@jrc.nl

${ }^{1}$ G. B. Arfken and H. J. Weber, Mathematical Methods For Physicists (Academic, San Diego, 2005).

${ }^{2}$ B. Davison and J. B. Sykes, Neutron Transport Theory (Clarendon, Oxford, 1957).

${ }^{3}$ G. I. Marchuk, Adjoint Equations and Analysis of Complex Systems (Kluwer, Secaucus, New Jersey, 1995).

${ }^{4}$ G. I. Bell and S. Glasstone, Nuclear Reactor Theory (Van Nostrand Reinhold, New York, NY, 1970), pp. 252-313.

${ }^{5}$ B. Wang, M. Goldstein, X. G. Xu, and N. Sahoo, "Adjoint Monte Carlo method for prostate external gamma beam treatment planning: An application to 3D patient anatomy," Phys. Med. Biol. 50, 923-935 (2005).

${ }^{6}$ F. C. Difilippo, "Forward and adjoint methods for radiotherapy planning," Med. Phys. 25, 1702-1710 (1998).

${ }^{7}$ F. C. Difilippo, M. Goldstein, B. A. Worley, and J. C. Ryman, "Adjoint Monte Carlo methods for radiotherapy treatment planning," Trans. Am. Nucl. Soc. 74, 14-16 (1996).

${ }^{8}$ M. Goldstein, "The adjoint Monte Carlo-a viable option for efficient radiotherapy treatment planning," Proceedings of the 19th Conference of the Israeli Nuclear Society 11-2 (1996).

${ }^{9}$ D. L. Henderson, S. Yoo, M. Kowalok, T. R. Mackie, and B. R. Thomadsen, "The adjoint method for the optimization of brachytherapy and radiotherapy patient treatment planning procedures using Monte Carlo calculations," Final Report for DOE-NEER Grant: DE-FG07-99ID13774 (2001).

${ }^{10}$ R. Jeraj and P. Keall, "Monte Carlo-based inverse treatment planning," Phys. Med. Biol. 44, 1885-1896 (1999).

11"MCNP-A general Monte Carlo N-particle transport code, version 4C," LA-13709-M , edited by J. F. Briesmeister (2000).

${ }^{12}$ B. L. Beers and V. W. Pine, "Functional expansion technique for Monte Carlo electron transport calculations" IEEE Trans. Nucl. Sci. 23, 18501856 (1976).

${ }^{13}$ R. F. Barth, J. A. Coderre, M. G. H. Vicente, and T. E. Blue, "Boron neutron capture therapy of cancer: Current status and future prospects," Clin. Cancer Res. 11, 3987-4002 (2005).

${ }^{14}$ Y. Nakagawa, K. Pooh, T. Kobayashi, Y. Sakurai, T. Kageji, S. Uyama, A. Matsumura, T. Yamamoto, and H. Kumada, "Clinical review of the Japanese experience with boron neutron capture therapy and a proposed strategy using epithermal neutron beams," J. Neuro-Oncol. 62, 87-99 (2003).

${ }^{15}$ M. L. Williams and W. W. Engle, Jr., "The concept of spatial channel theory applied to reactor shielding analysis," Nucl. Sci. Eng. 62, 92-104 (1977).

${ }^{16}$ J. C. Wagner, E. L. Redmond II, S. P. Palmtag, and J. S. Hendricks, "MCNP: Multigroup/adjoint capabilities," LA-12704, Los Alamos National Laboratory (1994).

${ }^{17}$ E. E. Lewis and W. F. Miller, Jr., Computational Methods of Neutron Transport (Wiley, New York, 1984).

${ }^{18}$ J. E. Hoogenboom, "Methodology of continuous-energy adjoint Monte Carlo for neutron, photon, and coupled neutron-photon transport," Nucl.
Sci. Eng. 143, 99-120 (2003).

${ }^{19} \mathrm{http}: / / \mathrm{www}-\mathrm{rsicc}$.ornl.gov/rsic-cgi-bin/enote.pl?nb=mcnp5\&action= view \&page $=116$; MCNP5 Notebook-page 116, F. Wasastjerna and T. Booth.

${ }^{20}$ A. Noel and H. S. Wio, "A new series-expansion approach in Monte Carlo: Application to neutron shielding," Ann. Nucl. Energy 11, 225-227 (1984).

${ }^{21}$ D. P. Griesheimer and W. R. Martin, "Monte Carlo based angular flux distribution with orthogonal function expansion," Trans. Am. Nucl. Soc. 89, 370-372 (2003).

${ }^{22}$ D. P. Griesheimer and W. R. Martin, "Two dimensional functional expansion tallies for Monte Carlo calculations," PHYSOR 2004-The Physics of Fuel Cycles and Advanced Nuclear Systems: Global Developments (2004).

${ }^{23}$ D. Legrady and J. E. Hoogenboom, "Midway Monte Carlo forwardadjoint coupling with Legendre polynomials," New Frontiers of Nuclear Technology: Reactor Physics, Safety and High-Performance Computing (2004).

${ }^{24}$ D. Legrady and J. E. Hoogenboom, "Visualization of space-dependency of responses of Monte Carlo calculations using Legendre polynomials," Proceedings PHYSOR 2004, Chicago, IL, April 25-29 (Elsevier Science, Chicago, 2004), pp. 1-7.

${ }^{25}$ D. P. Griesheimer, W. R. Martin, and J. P. Holloway, "Convergence properties of Monte Carlo functional/expansion techniques," J. Comput. Phys. 211, 129-153 (2006).

${ }^{26}$ J. P. Boyd, Chebyshev and Fourier Spectral Method (Dover, New York, 2000).

${ }^{27}$ R. C. Little and R. E. Seamon, "New MENDF5 and MENDF5G," Los Alamos Internal Memorandum X-6:RCL-86-412 (1986).

${ }^{28}$ JEF-2.2 Nuclear Data Library, JEFF Report 17, Paris, France (2000).

${ }^{29}$ K. A. Van Riper, "A CT and MRI scan to MCNP input conversion program," Radiat. Prot. Dosim. 115, 513-516 (2005).

${ }^{30}$ ICRU 46. Gamma, electron, proton and neutron interaction data for body tissues. International Commission on Radiation Units and Measurements, Bethesda, MD (1992).

${ }^{31}$ J. T. West III, SABRINA, "An interactive three-dimensional geometrymodeling program for MCNP," Los Alamos National Laboratory Report, LA10688M (1986).

${ }^{32}$ K. A. Van Riper, "New features in Sabrina," Proceedings of the Topical Meeting on Radiation Protection for our National Priorities, Spokane, WA, Sept. 17-21, (2000), pp. 316-323.

${ }^{33}$ EORTC protocol 11011 (version 2.0/February 2, 2004): Early phase II study on BNCT in metastatic malignant melanoma using the boron carrier BPA. European Organization for Research and Treatment of Cancer, Brussels (2004).

${ }^{34}$ R. H. Hardin, N. J. A. Sloane and W. D. Smith, "Tables of spherical codes with icosahedral symmetry," published electronically at http:// www.research.att.com/ njas/icosahedral.codes/. These tables are copyright R. H. Hardin, N. J. A. Sloane, and W. D. Smith, 1994 (2000). 